\title{
High pressure luminescence and time resolved spectra of $\mathrm{LiNbO}_{3}: \mathbf{P r}^{3+}$
}

\author{
A. Lazarowska ${ }^{1}, \mathrm{~S}$. Mahlik $^{1}$, M. Grinberg ${ }^{1 *}$, M. Malinowski ${ }^{2}$ \\ ${ }^{1}$ Institute of Experimental Physics, University of Gdańsk, Wita Stwosza 57, 80-952 Gdańsk, Poland, \\ ${ }^{2}$ Institute of Micro- and Optoelectronics, Warsaw University of Technology, Koszykowa 75, \\ 00-662 Warsaw, Poland
}

Received May 18, 2011; accepted June 06, 2011; published June 30, 2011

\begin{abstract}
Emission, luminescence excitation spectra and photoluminescence spectra at high pressure of $\mathrm{LiNbO}_{3}$ crystal doped with $0.5 \% \mathrm{Pr}^{3+}$ are presented. Hydrostatic pressure up to $135 \mathrm{kbar}$ was applied with a diamond anvil cell (DAC). We observed the small red shift of the luminescence related to ${ }^{1} \mathrm{D}_{2} \rightarrow{ }^{3} \mathrm{H}_{4}$ transition equal to $0.08 \mathrm{~nm} / \mathrm{kbar}$. Additionally, at higher pressure, the ${ }^{1} \mathrm{D}_{2}$ emission was considerably quenched and its lifetime was shortened. This was explained as being due to the decrease of the energy of the exciton bound to the $\operatorname{Pr}^{3+}$ ion with increasing pressure and nonradiative depopulation of the ${ }^{1} \mathrm{D}_{2}$ state.
\end{abstract}

Recently, there has been a rapid growth of interest in the study of spectral properties of $\mathrm{RE}$ doped $\mathrm{LiNbO}_{3}$ crystals, [1-11]. The interest in $\operatorname{Pr}^{3+}$ ion is due to its ability to emit V-UV photons, due to interconfigurational $4 \mathrm{f}^{1} 5 \mathrm{~d}^{1} \rightarrow 4 \mathrm{f}^{2}$ transitions, and green and red photons related to the intraconfigurational transitions ${ }^{3} \mathrm{P}_{0} \rightarrow{ }^{3} \mathrm{H}_{\mathrm{J}}$ and ${ }^{1} \mathrm{D}_{2} \rightarrow{ }^{3} \mathrm{H}_{\mathrm{J}}$ in the $4 \mathrm{f}^{2}$ electronic configuration, respectively. In oxides, we have mainly observed the VIS and IR emission related to the ${ }^{3} \mathrm{P}_{0} \rightarrow{ }^{3} \mathrm{H}_{\mathrm{J}}$ and ${ }^{1} \mathrm{D}_{2} \rightarrow{ }^{3} \mathrm{H}_{\mathrm{J}},{ }^{1} \mathrm{D}_{2} \rightarrow{ }^{3} \mathrm{~F}_{\mathrm{J}}$, and ${ }^{1} \mathrm{D}_{2} \rightarrow{ }^{1} \mathrm{G}_{4}$ transitions, respectively, whereas in $\mathrm{LiNbO}_{3}: \mathrm{Pr}^{3+}$, only the red and IR luminescence related to transitions from the ${ }^{1} \mathrm{D}_{2}$ state is observed $[10,11]$. The lack of the ${ }^{3} \mathrm{P}_{0} \rightarrow{ }^{3} \mathrm{H}_{\mathrm{J}}$ emission in $\mathrm{Pr}^{3+}$ in $\mathrm{LiNbO}_{3}$ has been related to the existence of the $\operatorname{Pr}^{3+}$ trapped exciton (PTE) [12].The first approach to the high pressure spectroscopy of $\mathrm{LiNbO}_{3}: \mathrm{Pr}^{3+}$ was demonstrated in our previous papers $[13,14]$. In paper [13] we focused on structure analysis of sharp line emission. We described PTE in the $\mathrm{LiNbO}_{3}$ as a system where the $\operatorname{Pr}^{3+}$ ion captured a hole ( forming the $\mathrm{Pr}^{2+}$ ) which bound an electron at Rydberg states, [14]. In $\mathrm{LiNbO}_{3}$ the energy of PTE is a little bit smaller than the energy of the ${ }^{3} \mathrm{P}_{0}$ state of the $\mathrm{Pr}^{3+}$. In paper [14] it was found that pressure diminishes the energy of PTE that quenches the $\operatorname{Pr}^{3+}$ luminescence. Additionally, it was found that at high pressure under $\mathrm{CW}$ Ar laser excitation with $488 \mathrm{~nm}$, the ${ }^{1} \mathrm{D}_{2} \rightarrow{ }^{3} \mathrm{H}_{4}$ luminescence was accompanied by broad band emission with its maximum at the wavelength longer than $900 \mathrm{~nm}$. This luminescence was tentatively attributed to the recombination of PTE. Although the existence of PTE in $\mathrm{LiNbO}_{3}: \mathrm{Pr}^{3+}$ has been proved, the effect of radiative recombination of PTE was

*E-mail: fizmgr@univ.gda.pl not well evidenced. Since at high pressure and room temperature the emission of $\mathrm{LiNbO}_{3}: \mathrm{Pr}^{3+}$ is very week, the observed broad band luminescence could be also an artifact related to DAC emission. Therefore, we decided to repeat the experiments using new more sensitive equipment which, additionally, allows to control simultaneously the luminescence and luminescence kinetics.

The $\mathrm{LiNbO}_{3}$ doped with $0.5 \mathrm{at} \% \mathrm{Pr}^{3+}$ and $0.8 \mathrm{at} \% \mathrm{Yb}^{3+}$ was grown by the Czochralski method in I.T.M.E. Laboratory in Warsaw. The details of sample preparation were described in our previous paper [13].

Measurements of the luminescence excitation spectrum were made using a spectrofluorimeter FluoroMax-4P TCSPC produced by Horiba. The excitation source in this system was a 150 Watts Xenon lamp which makes it possible to measure luminescence excitation in the 220 $850 \mathrm{~nm}$ range. Fluorescence intensity is measured by using a monochromator and a photomultiplier R928 Sideon

The experimental setup for luminescence kinetics is a laser system which consists of a PL 2143 A/SS laser with $10 \mathrm{~Hz}$ frequency and the parametric optical generator PG $401 / \mathrm{SH}$. The laser generates $30 \mathrm{ps}$ pulses of the $355 \mathrm{~nm}$ wavelength and the pump PG generator produces light pulses ranging from 220 to $2200 \mathrm{~nm}$. The output energy at the $320 \mathrm{~nm}$ wavelength was $20 \mu \mathrm{J}$. The emission signal was analyzed by the spectrometer 2501S (Bruker Optics) equipped with a 50 grooves $/ \mathrm{mm}$ grating and a Hamamatsu Streak Camera model C4334-01 with a final spectral resolution of about $0.47 \mathrm{~nm}$. Time resolved luminescence spectra were collected by integration of streak camera pictures over time intervals, whereas luminescence decays were collected by the integration of streak camera pictures over the wavelength intervals.

High hydrostatic pressure was applied in a diamond anvil cell (DAC) Merrill Bassett type with silicone oil (polydimethylsiloxane) as a pressure-transmitting medium. The pressure was measured by the shift of the $\mathrm{R}_{1}$ photoluminescence line of ruby.

Ambient pressure excitation spectrum of emission monitored at $618 \mathrm{~nm}\left(16181 \mathrm{~cm}^{-1}\right)$ is presented in Fig. 1. 


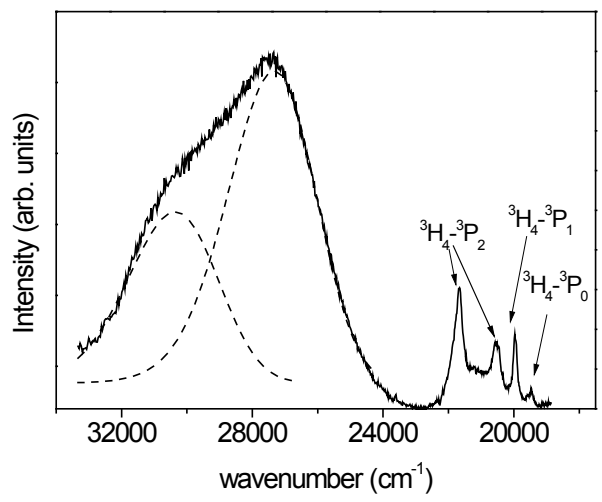

Fig.1. Ambient temperature luminescence excitation spectrum monitored at $16181 \mathrm{~cm}^{-1}$ of the $\mathrm{LiNbO}_{3}: \mathrm{Pr}^{3+}$.

The spectrum consists of sharp lines in the spectral region $19000 \mathrm{~cm}^{-1}-22000 \mathrm{~cm}^{-1}$ related to ${ }^{3} \mathrm{H}_{4} \rightarrow{ }^{3} \mathrm{P}_{0},{ }^{3} \mathrm{P}_{1},{ }^{3} \mathrm{P}_{2}$ transitions and the broad band extended between $24000 \mathrm{~cm}^{-1}$ and $34000 \mathrm{~cm}^{-1}$. The broad band structure was decomposed into two Gaussian bands peaked at 30400 $\mathrm{cm}^{-1}$ and $27360 \mathrm{~cm}^{-1}$, related to charge transfer (CT) transition and creation of PTE. From luminescence excitation spectrum presented in Fig. 1 one notices that the sample is more effectively excited through the band in $24000 \mathrm{~cm}^{-1}-32000 \mathrm{~cm}^{-1}$ region than through the $\mathrm{f}-\mathrm{f}$ transition in the spectral region $19000 \mathrm{~cm}^{-1}-22000 \mathrm{~cm}^{-1}$.

In Fig. 2(a) the luminescence spectra of $\mathrm{LiNbO}_{3}: \mathrm{Pr}^{3+}$ obtained at different pressures are presented. The sample was excited with a wavelength of $320 \mathrm{~nm}$ (Yag: $\mathrm{Nd}$ pulsed laser and OPG system). All spectra were recorded at room temperature.
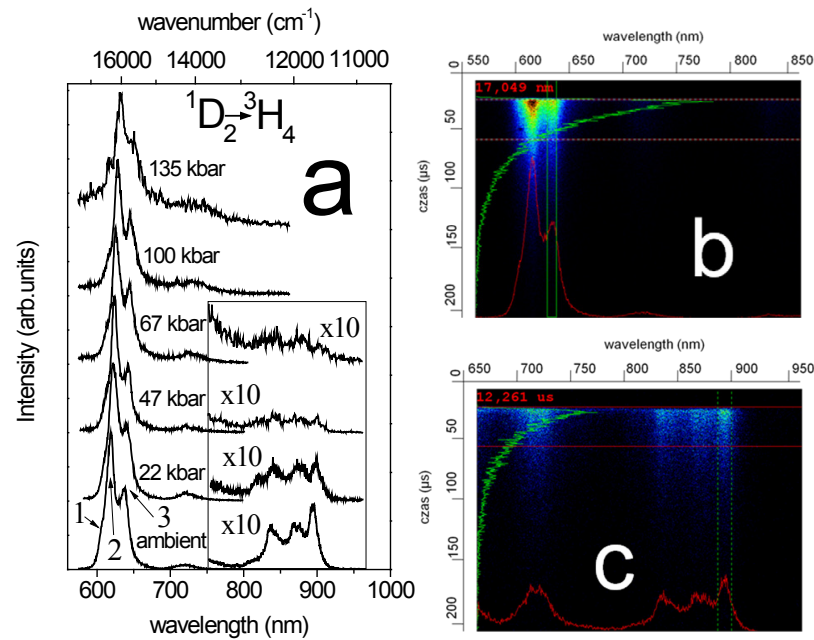

Fig. 2. (a) Time resolved luminescence spectra under pressure: time acquisition $0-200 \mu \mathrm{s}$, emission from ${ }^{1} \mathrm{D}_{2}$ state. (b) Streak camera picture for spectral region $550 \mathrm{~nm}-850 \mathrm{~nm}$. (c) Streak camera picture for the spectral region $650 \mathrm{~nm}-950 \mathrm{~nm}$.

Actually, in Fig. 2(a) time resolved luminescence spectra are presented, where acquisition was done in time from 0 to $200 \mu$ s after excitation.
The spectra in Fig. 2(a) consist of sharp lines related to ${ }^{1} \mathrm{D}_{2} \rightarrow{ }^{3} \mathrm{H}_{4}$ transition in the spectral region $680 \mathrm{~nm}-580 \mathrm{~nm}$ $\left(14700 \mathrm{~cm}^{-1}-17250 \mathrm{~cm}^{-1}\right)$ and the sharp lines in the region $910 \mathrm{~nm}-700 \mathrm{~nm} \quad\left(11000 \mathrm{~cm}^{-1}-13300 \mathrm{~cm}^{-1}\right) \quad$ related to ${ }^{1} \mathrm{D}_{2} \rightarrow{ }^{3} \mathrm{~F}_{2,3,4},{ }^{3} \mathrm{H}_{6}$ transitions.

One can recognize three lines related to the ${ }^{1} \mathrm{D}_{2} \rightarrow{ }^{3} \mathrm{H}_{4}$ transition; $608.1 \mathrm{~nm}, 619.4 \mathrm{~nm}$ and $637.8 \mathrm{~nm}$ labelled in Fig. 2(a) by numbers 1, 2 and 3, respectively.

The selected original pictures obtained from the streak camera at ambient pressure are presented in Fig. 2(b)-(c). In Fig. 2(b) we have the spectrum obtained in the region from 550nm to 850nm, where the ${ }^{1} \mathrm{D}_{2} \rightarrow{ }^{3} \mathrm{H}_{4}$ luminescence is detected. Figure 2(c) obeys the spectral region between $650 \mathrm{~nm}$ and $950 \mathrm{~nm}$, which corresponds to weaker luminescence related to the ${ }^{1} \mathrm{D}_{2} \rightarrow{ }^{3} \mathrm{~F}_{2,3,4},{ }^{3} \mathrm{H}_{6}$ transitions. Both pictures were collected in the time scale after pulse (30ps) excitation from 0 to $200 \mu \mathrm{s}$ after pulse. In Figs. 2(b) and (c) the red curves show the spectra, whereas the green curves show the luminescence decays.

One notices that both emissions decay in the same way, which confirms the assumption that they both come from the same initial state; the ${ }^{1} \mathrm{D}_{2}$. We have not obtained any broad band luminescence in the red spectral region for all considered pressure at room temperature, which could be attributed to PTE recombination.

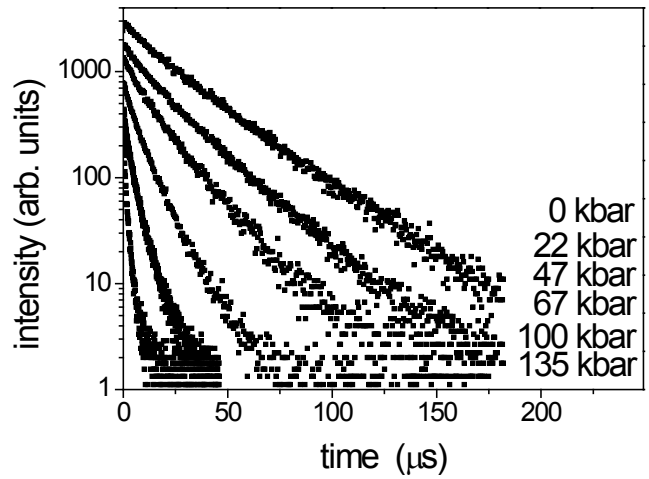

Fig. 3. Luminescence decay curves under different pressures for luminescence monitored at $16181-15674 \mathrm{~cm}^{-1}(618-638 \mathrm{~nm})$ that corresponds to ${ }^{1} \mathrm{D}_{2}-{ }^{3} \mathrm{H}_{4} \operatorname{Pr}^{3+}$ emission.

When pressure increases, the emission lines shift mainly to the red region. The respective pressure shifts of the lines related to ${ }^{1} \mathrm{D}_{2} \rightarrow{ }^{3} \mathrm{H}_{4}$ transition are $0.081 \mathrm{~nm} / \mathrm{kbar}$, $0.089 \mathrm{~nm} / \mathrm{kbar}$ and $0.080 \mathrm{~nm} / \mathrm{kbar}$ for the lines peaked at $608.1 \mathrm{~nm}, 619.4 \mathrm{~nm}$ and $637.8 \mathrm{~nm}$, respectively. When pressure increases, the intensity of luminescence decreases. This effect is accompanied by faster luminescence decay. The decay of luminescence related to ${ }^{1} \mathrm{D}_{2} \rightarrow{ }^{3} \mathrm{H}_{4}$ transition obtained at different pressures is presented in Fig. 3. The effective emission decay time decreases from $25 \mu \mathrm{s}$ at ambient pressure to $3 \mu \mathrm{s}$ at $135 \mathrm{kbar}$. 
The shorter luminescence decay is related to the nonradiative dexciation of the ${ }^{1} \mathrm{D}_{2}$ state of $\operatorname{Pr}^{3+}$ through the PTE state. This process is described by configurational coordinate diagrams presented in Figs. 4(a) and (b).
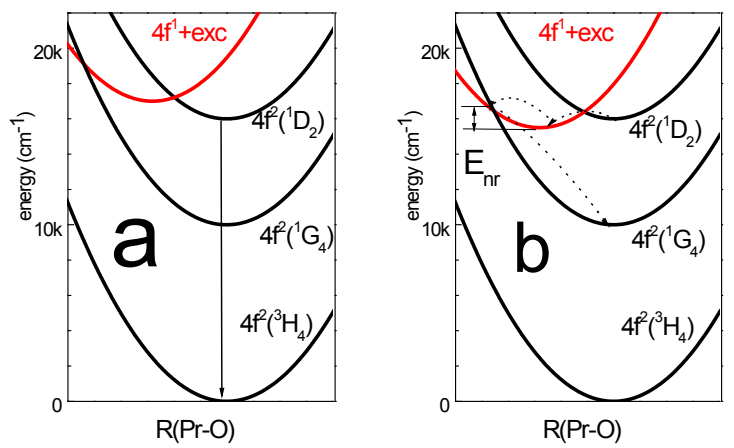

Fig. 4. Configurational coordinate diagrams representing the energetic structure of $\mathrm{Pr}^{3+}$ ion and PTE. At ambient pressure and energy of the ${ }^{1} \mathrm{D}_{2}$ state below energy of PTE (a) and at high pressure and the ${ }^{1} \mathrm{D}_{2}$ state above energy of PTE (b).

The black parabolas in the diagrams represent the electronic energies of the ground state of the $\operatorname{Pr}^{3+}$ : the ${ }^{3} \mathrm{H}_{4}$ state, the luminescent state ${ }^{1} \mathrm{D}_{2}$ and the ${ }^{1} \mathrm{G}_{4}$ state, which energy is just below of the ${ }^{1} \mathrm{D}_{2}$. The red parabolas represent the electronic energy of PTE states. The abscissa axis in Figs. 4(a) and (b) represents the average distance between $\mathrm{Pr}^{3+}$ and $\mathrm{O}^{2-}$ ions; $R$. Since the hole captured at $\mathrm{Pr}^{3+}$ attracts the $\mathrm{O}^{2-}$ ligands, minimum of the parabola corresponding to the PTE appears for the smaller quantity of $R$ than minima of the parabolas representing the localized states of the $\mathrm{Pr}^{3+}$. The diagram in Fig. 4(a) corresponds to ambient pressure. Here PTE state is above the emitting state of $\operatorname{Pr}^{3+} ;{ }^{1} \mathrm{D}_{2}$ state and radiative transition from ${ }^{1} \mathrm{D}_{2}$ state to the ground state ${ }^{3} \mathrm{H}_{4}$ is possible (transition represented by solid arrow). In Fig. 4(b) a high pressure situation is presented. Here the PTE state is below the ${ }^{1} \mathrm{D}_{2}$ state and emission from the ${ }^{1} \mathrm{D}_{2}$ state is quenched. The deexcitation of the $\operatorname{Pr}^{3+}$ takes place by the nonradiative processes through the PTE state. In Fig. 4(b) the nonradiative deexciation pathway is presented by dashed arrows. One notices that nonradiative depopulation of the PTE state is much faster when respective activation energy $E_{n r}$ is smaller. The effective luminescence decay time depends on radiative depopulation rate, $p_{r}$ and nonradiative depopulation rate $p_{n r}$, while

$$
\frac{1}{\tau_{e f f}}=p_{r}+p_{n r}
$$

and the nonradiative depopulation rate $p_{n r}$ increases when the energy of PTE decreases and causes energy $E_{n r}$ to decrease [see Fig. 4(b)].
As a result, luminescence decays faster and the respective luminescence decay time is shorter. For pressures higher than $135 \mathrm{kbar}$, no luminescence related to $\operatorname{Pr}^{3+}$ was observed.

In conclusion, the photoluminescence spectra of $\mathrm{LiNbO}_{3}: \mathrm{Pr}^{3+}$ obtained at different pressures are presented. At ambient conditions one observed effective luminescence related to radiative transitions from the ${ }^{1} \mathrm{D}_{2}$ state of $\mathrm{Pr}^{3+}$. When pressure increases, the intensity of luminescence decreases and simultaneously the emission lifetime is shortened. These effects were attributed to the nonradiative recombination of PTE which energy decreases with increasing pressure. For pressure larger than $135 \mathrm{kbar}$, the luminescence of $\operatorname{Pr}^{3+}$ was not observed.

This paper was supported by the Polish Ministry of Science and Higher Education with Grants active in the years 2009-2011. One of the authors (A.L.) was supported within the International $\mathrm{PhD}$ Project (MPD/2009-3/4) financed by the Foundation for Polish Science.

\section{References}

[1] H. Laro, M. Voda, F. Jaque, J.E. Munoz-Santiuste, J. Garcia-Sole, J. Appl. Phys. 77, 5929 (1995)

[2] A. Lorenzo, L.E. Bausa, J. Garcia-Sole, Phys. Rev. B. 51, 16643 (1995).

[3] A. Lorenzo, E. Camarllo, H.S. Maurrieta, S. Alvarez, V.G. Hemandez, M. Voda, F. Jaque, J. Garcia-Sole, Opt. Matter. 5, 251 (1996).

[4] M. Malinowski, P. Myziak, R. Piramidowicz, I. Pracka, T. Łukasiewicz, B. Surma, S. Kaczmarek, K. Kopczyński, Z. Mierczyk, Acta Phys. Pol. 90, 181 (1996).

[5] A. Lorenzo, L.E. Bausa, J.A. Sanz, J. Garcia-Sole, J. Phys.: Cond Matter 8, 5781 (1996)

[6] J. Amin, B. Dusardier, T. Schweizer, M. Hempsead, J. Lumin. 69,17 (1966).

[7] L. Nunez F. Cusso, J. Phys.: Cond. Matter. 5, 5301 (1993).

[8] Z. Łuczyński, T. Łukasiewicz, I. Pracka, B. Surma, M. Malinowski, J. Appl. Spectr. 62, 66 (1995).

[9] A. Lorenzo, H. Jaffrezic, B. Roux, G. Boulon, L.E. Bausia, J. Garcia-Sole, Phys. Rev. B 52, 6278 (1995).

[10] R. Piramidowicz, I. Pracka, W. Woliński, M. Malinowski, J. Phys.:Cond Matter 12, 709 (2000).

[11] J.E. Munoz-Santiuste, A. Lorenzo, L.E. Bausa, J. Garcia-Sole, J. Phys.: Cond Matter 10, 7653 (1998).

[12] D.S. McClure, C. Pedrini, Phys. Rev. B 32, 8465 (1985).

[13] W. Gryk, B. Kukliński, M. Grinberg, M. Malinowski, J. Alloy. Comp. 380, 230 (2004).

[14] W. Gryk, D. Dyl, M. Grinberg, M. Malinowski, Phys. Stat. Sol. C 2, $188(2005)$ 